\title{
Ultrasound Imaging of the Menstrual Cycle
}

\author{
${ }^{1}$ Herbert F Janssen, ${ }^{2}$ Sanja Plavsic Kupesic
}

${ }^{1}$ Professor of Physiology, Department of Medical Education and Department of Orthopedic Surgery and Rehabilitation, Paul L Foster School of Medicine, Texas Tech University Health Sciences Center, 5001 El Paso Drive, El Paso, Texas 79905, USA

${ }^{2}$ Professor and Clinical Professor of Obstetrics and Gynecology and Radiology, Department of Medical Education, Paul L Foster School of Medicine, Texas Tech University Health Sciences Center, 5001 El Paso Drive, EI Paso, Texas 79905, USA

Correspondence: Herbert F Janssen, Professor of Physiology, Department of Medical Education and Department of Orthopedic Surgery and Rehabilitation, Paul L Foster School of Medicine, Texas Tech University Health Sciences Center, 5001 El Paso Drive, El Paso, Texas 79905, USA, e-mail: herb.janssen@ttuhsc.edu

\begin{abstract}
The menstrual cycle is nature's repeating preparation for pregnancy. Each month, the uterine lining develops in anticipation of implantation by a fertilized ovum. If this occurs, hormones from the reproductive organs stabilize the vascular tissue of the endometrium which provides an environment for the developing embryo. If the awaited implantation does not occur, the endometrium is sloughed due to a rapid drop in circulating levels of progesterone. This process starts during the early teenage years of a female's development (menarche) and repeats 400-500 times over the next 40 years. Normally, this process is interrupted only by pregnancy; however, other factors can certainly influence both the cycle length, volume, and the duration of each menses. Ultrasound imaging provides a noninvasive method to visualize the internal reproductive organs, the endometrium, and the embryo if implantation occurs. This relatively inexpensive evaluation provides an easy and cost effective method to examine potential problems associated with the female reproductive organs. Advances in computer software and hardware coupled with new ultrasound equipment has provided exciting 3-D and 4-D images that generate near life-like images. In the guiding hand of a trained physician or sonographer, ultrasound enhances diagnostic capability and gives reassurance to the patient.
\end{abstract}

Keywords: Menstrual cycle, endometrium, ultrasound, hypothalamic-pituitary-gonadal axis, pregnancy.

\section{INTRODUCTION}

The female reproductive system is a uniquely beautiful interaction of anatomy, biochemistry, histology, and physiology. Many biochemical and histological events occur unnoticed by the fertile female; however, the monthly menstrual cycle is a repeating reminder that she is capable of conceiving and developing new life. This cycle develops the endometrium to receive the fertilized ovum. When, as is most often the case, the fertilized ovum never arrives, the uterus, unwavering in its mission, starts anew to prepare for the potential of implantation during the next monthly cycle. When implantation occurs, the uterus provides a safe environment where the embryo can develop and mature until he or she is ready for delivery. Even then, the uterus quickly recovers and can be ready to accept a new fertilized ovum within several months after delivery.

The initial menstrual cycle (menarche) usually occurs at approximately 13 years of age. The cycles normally continue, interrupted only by pregnancy, until menopause. During the 40 or so intervening years, the normal process repeats some 400 to 500 times. ${ }^{1}$ While seemingly wasteful, this regenerative process is nature's way of ensuring that a new, fresh home for the fertilized ovum awaits and if the opportunity presents itself is ready to fulfill its most important task of perpetuating human existence.

The normal anatomy of the pelvis and reproductive organs are illustrated in the figures below (Figs 1 and 2). The mission of these structures is interconnected by both their anatomical location as well as their physiological function. Their activity is controlled in large part by hormones released from the pituitary. These hormones interact with estrogen and progesterone to control the development and maturation of the ovum, the physiology of the uterus, and the menstrual cycle. The anatomy as well as the physiology will be explored in more detail in the later sections.

These same structures have been labeled in the ultrasound images below. Images of the deep structures are typically presented using a transabdominal view obtained with a lower frequency probe that allows greater penetration. Images can also be obtained using transvaginal and transrectal probes. These probes allow the ultrasonographer to use a higher frequency ultrasound probe capable of obtaining images with improved resolution. 


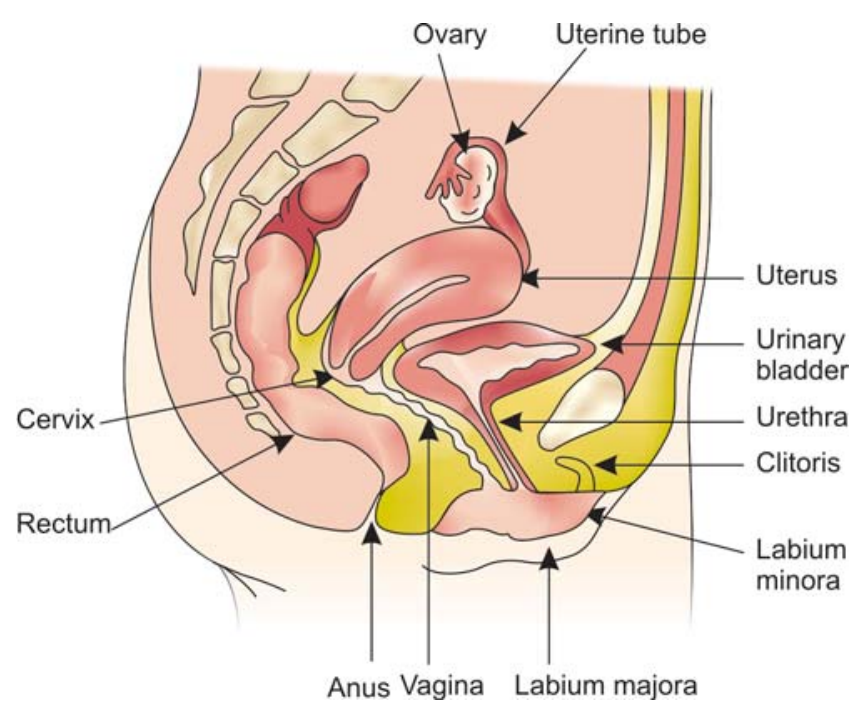

Fig. 1: Anatomy of the female pelvis. ${ }^{1}$

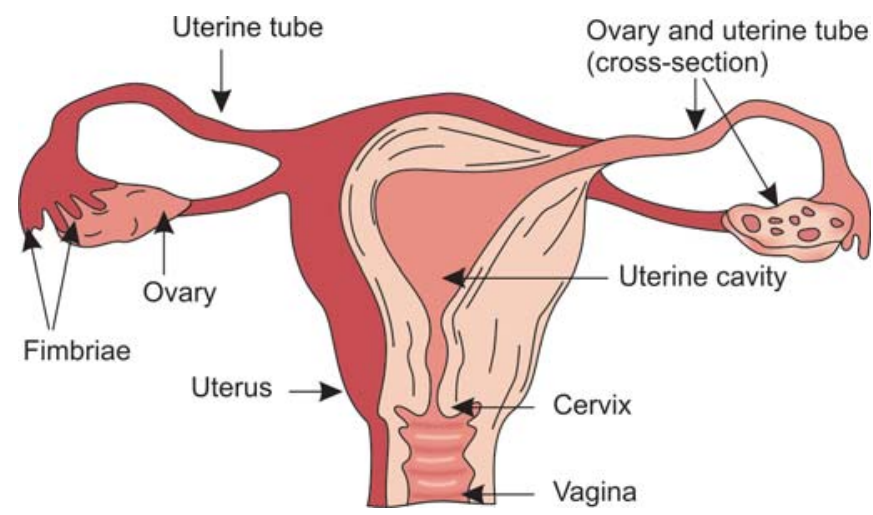

Fig. 2: Expanded view of the female reproductive organs. The anatomical arrangement and the structures coupled with hormonal control provide an interconnection that is necessary for the normal reproductive process. ${ }^{1}$

\section{HORMONAL INTERACTIONS}

The menstrual cycle and pregnancy are controlled by a complex interaction of hormones. The hypothalamus secretes gonadotropin-releasing hormone $(\mathrm{GnRH})$ which stimulates the release of follicle-stimulating hormone (FSH) and luterinizing hormone ( $\mathrm{LH})$ from the pituitary. The ovarian hormones estrogen and progesterone are released by the ovaries in response to the two gonadotropic hormones arising from the pituitary. The interaction continues well beyond this initial control. The released hormones provide feedback to the brain and pituitary to retain the delicate balance that must be preserved. As would be expected, the feedback messages also signal almost instantly when implantation occurs and pregnancy is started.

\section{THE HYPOTHALAMUS}

The hierarchy of hormonal control originates in the brain. The release of GnRH for the arcuate nuclei of the mediobasal region of the hypothalamus is in pulsatile bursts emitted in 90 minute repeating cycles. This burst of GnRH is thought to be controlled by neuronal activity in the hypothalamus. As might be expected, this pulsatile release produces a similar phasic response in the release of LH. The pulsatile nature of the hormonal release is necessary for normal function. It has been clearly demonstrated that continuous administration of GnRH inhibits the normal release of LH. The figure below (Fig. 3) depicts this pulsatile release of LH which corresponds to neural activity in the brain. ${ }^{2}$

This cycle is inherent in the GnRH neurons but can be regulated by other outside influences. These outside influences include dopamine, norepinephrine, and neuropeptide $Y{ }^{3}$ Studies in animals have demonstrated that continuous infusion of GnRH renders the hormone control of LH and FSH from the pituitary ineffective. ${ }^{4}$ Although GnRH controls the release of both pituitary hormones, research has demonstrated that different GnRH pulse frequencies produce different release patterns. For example, a pulse each hour preferentially increases LH release while a slower rate of one pulse every 3 hours produces a greater effect on FSH. While the significance of this is unknown, it does illustrate the complex nature of the hormonal control. ${ }^{5}$

The release of GnRH from the hypothalamus is under both negative (-) and positive (+) feedback control from estrogen and progesterone. Inhibin, a hormone secreted by the granulosa cells of the corpus luteum, also provides a powerful (-) feedback to down regulate the production of FSH and to a lesser degree LH at the end of the monthly cycle. While a complete understanding of the process is

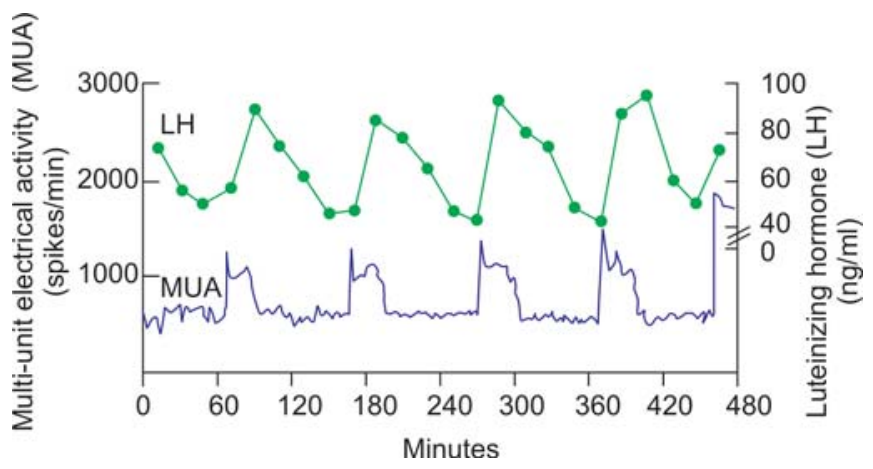

Fig. 3: The pulsatile release of $\mathrm{GnRH}$ from the hypothalamus occurs on a repeating 90 minutes cycle. Continuous infusion of $\mathrm{GnRH}$ has been shown in animals to be ineffective in stimulating the normal release of $\mathrm{LH}$ and $\mathrm{FSH}^{1}$ 
lacking, it is reasonable to assume that many factors, including psychological moods can alter or influence the female reproductive cycle.

\section{THE PITUITARY}

The gonadotropins, LH and FSH are synthesized and secreted from gonadotroph cells located in the anterior pituitary. Their release is stimulated by GnRH binding to Type I receptors. Other receptor types have been identified in nonhuman primates but their role has not been fully described. The plasma concentration of the two pituitary hormones varies depending on their rate of release from the pituitary and their rate of removal from the plasma. The gonadotropins are released from the pituitary during the monthly cycle at a frequency and amplitude dependent both on the time in the cycle and the phasic nature of GnRH release from the hypothalamus. The cyclic nature and magnitude can be easily determined for LH. Such a rhythm for FSH is more difficult to evaluate due to its longer half-life in the plasma. ${ }^{6}$ This increased half-life is due primarily to the carbohydrate residues that decrease the hormones removal from the plasma. $^{7}$

\section{THE OVARIES}

The release of the LH and FSH from the pituitary is also greatly influenced by estrogen and progesterone released from the ovaries. This feedback can be either negative (-) or positive (+) depending on the concentration of both hormones. This complex feedback mechanism provides a unique control system that regulates the endometrium and in preparation for the potential of receiving a fertilized ovum. The two pituitary hormones also play a pivotal role in the development of the follicles in the ovaries.

Inhibin is released from the granulosa cells and appears as two separate subtypes - inhibin A and inhibin B. Evidence exists that inhibin is both stimulated by FSH and plays a role in the inhibition of this gonadotropin hormone. This reciprocal relation is thought to play a significant role in the regulation of the normal menstrual cycle. This appears to be particularly true at the end of the monthly cycle where FSH and to a lesser degree LH is downregulated by inhibin. ${ }^{1}$

Under the control of mainly FSH, 8-12 follicles undergo accelerated growth. Finally, one of these out-grows the others in preparation for final maturation and release. On day 14 of the menstrual cycle, a surge of LH stimulates release of the chosen ovum. Selection of the dominant follicle from the cohort of developing follicles occurs normally between day 5 and 7 of the menstrual cycle, but its dominance is not apparent sonographically until cycle day 8-12 (Figs 4 to 6). A decline in the follicle stimulating hormone (FSH), which occurs in the late follicular phase, is responsible for the selection of the single, most mature follicle. Follicles with fewer FSH receptors on their surfaces become atretic. These subordinate antral follicles rarely reach more than $14 \mathrm{~mm}$ in diameter and therefore, any follicle exceeding $14 \mathrm{~mm}$ is usually the dominant follicle. At the time the follicle ruptures, it usually reaches $20 \mathrm{~mm}$ in mean diameter. Size alone is not a good predictor of impending ovulation, but careful sonographic visualization of the maturing follicle may show the development of a sonolucent halo around the follicle, which reflects the preovulatory change in response to the LH surge and is seen within 24 hours before ovulation (Fig. 7). Detection of the cumulus

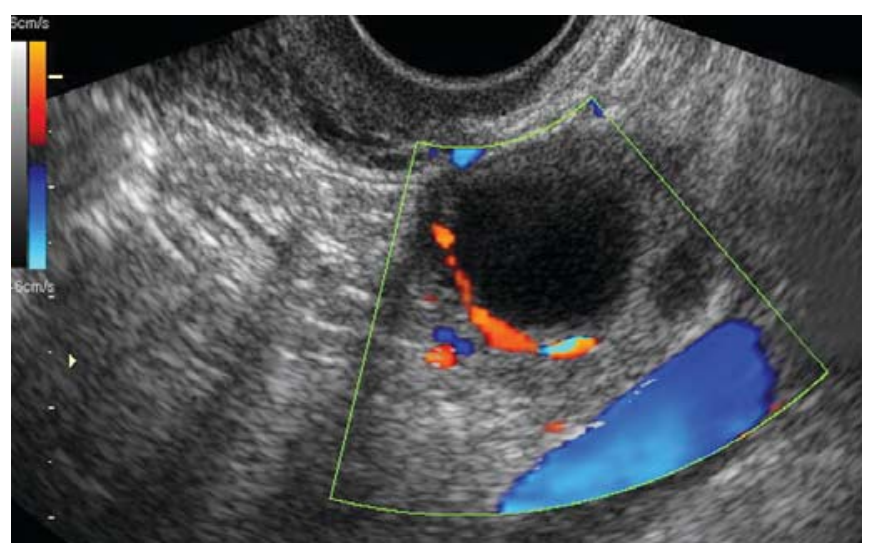

Fig. 4: Transvaginal color Doppler scan demonstrating prominent ring of angiogenesis surrounding the dominant follicle

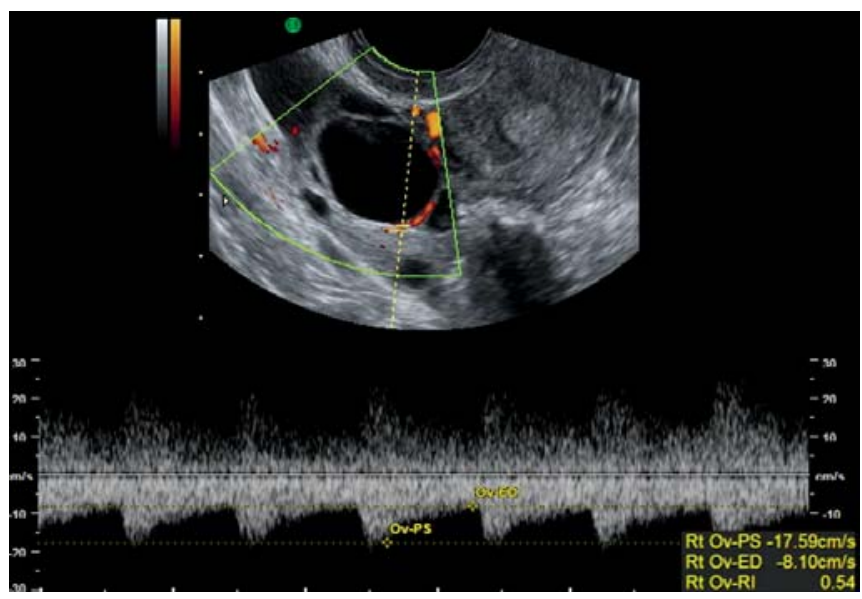

Fig. 5: Pulsed Doppler waveform analysis of the follicular vessels obtained during the phase of the follicular growth shows a resistance index of 0.54 


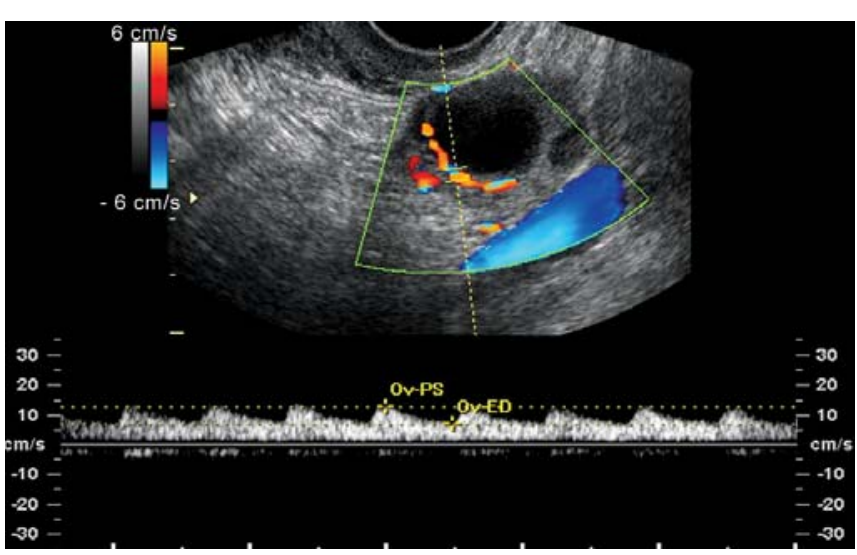

Fig. 6: The resistance index equals 0.45 as ovulation approaches, which indicates vasodilatation of the follicular capillaries
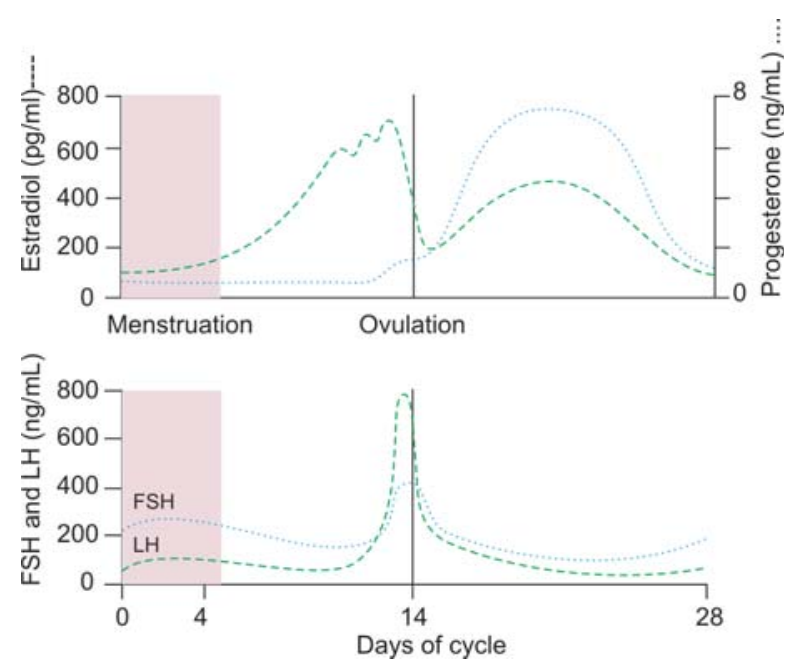

Fig. 7: The changes in hormone levels is Illustrated in relationship to the menstrual cycle ${ }^{1}$

oophorus within the follicle is a sign of imminent ovulation (Figs 8 and 9). Blood flow velocity increases before ovulation, and it is considered that, apart from hormonal factors, angiogenesis is also involved (Fig. 10).

Once released, it travels down the fallopian tubes. If it is fertilized during this trip, it will be ready to implant in the expectant lining of the uterus that had been thickening in preparation for the event. If it remains unfertilized, it will pass from the uterus and the thickened lining will be discarded in the menstrual bleeding that signals a start in the repeating monthly cycle if pregnancy has not occurred. If implantation had occurred and pregnancy was initiated, hormonal signals would have maintained the uterine wall which would become home for the developing embryo.

\section{THE ENDOMETRIUM}

The endometrium or lining of the uterus thickens in preparation to receive the fertilized ovum. This occurs in

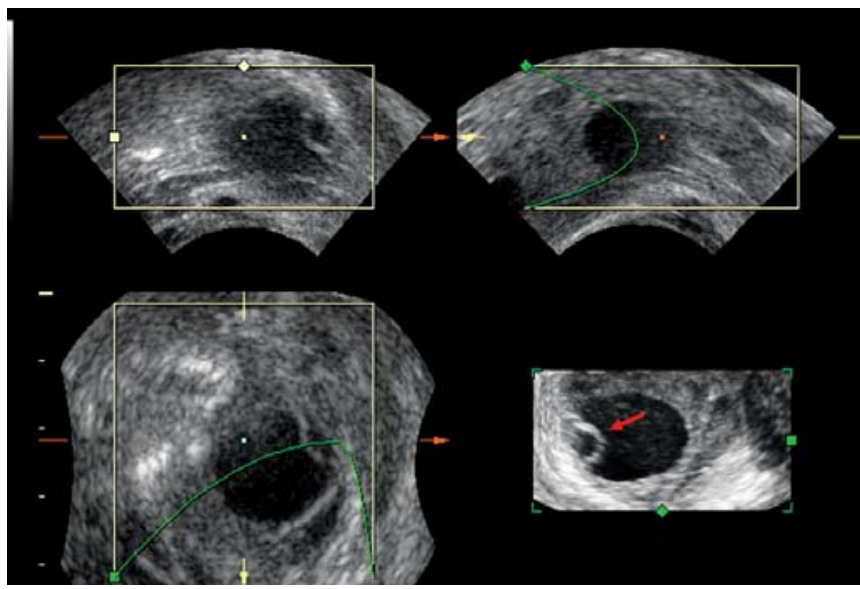

Fig. 8: Three-dimensional sonogram of the preovulatory follicle. Note cumulus oophorus bulging from the inner wall of the follicle (red arrow)

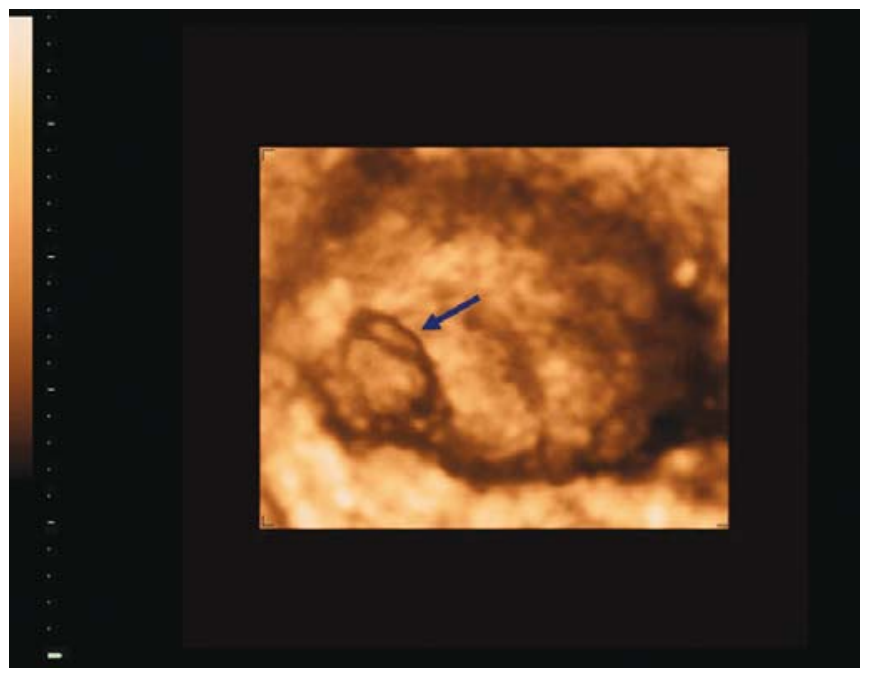

Fig. 9: Surface rendering of the preovulatory follicle containing cumulus oophorus (arrow)

several phases. The endometrium is, for the most part, absent at the end of the monthly cycle having been sloughed during the menstrual phase. Slowly it starts to rebuild during the proliferative phase (Fig 11). Stimulated by estrogen released from the ovaries, the endometrium rebuilds rapidly developing new blood vessels to feed the stromal cells that proliferate rapidly. As a result, the structure reaches a thickness

4 to $7 \mathrm{~mm}$ by the time of ovulation on the 14th day of the cycle. ${ }^{1,6}$

Ultrasound can be used to observe the development of the endometrium as it develops during the course of the menstrual cycle. As mentioned previously, the endometrium is almost absent at the completion of a normal menses (Fig. 12A); however, it quickly grows under the influence 


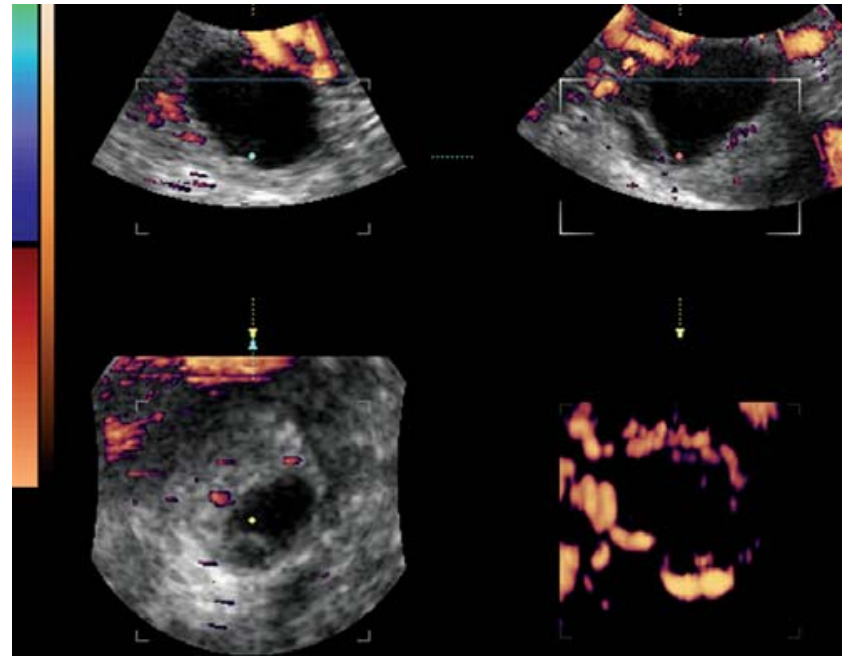

Fig. 10: Three-dimensional power Doppler scan of the preovulatory follicle demonstrating the ring of angiogenesis

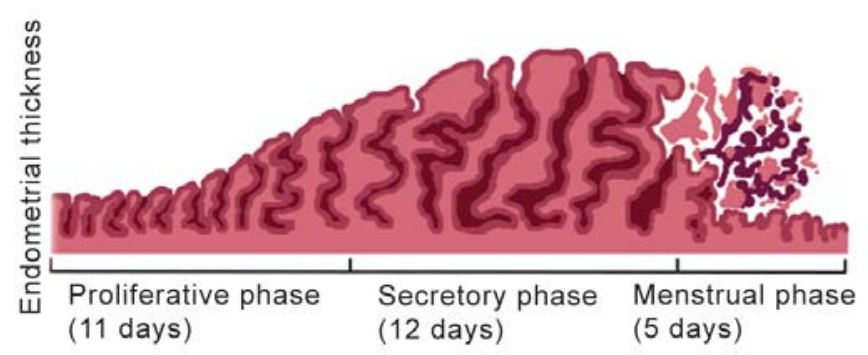

Fig. 11: The endometrium or lining of the uterus thickens in preparation to receive the fertilized ovum ${ }^{1}$

of estrogen released from the ovaries (Fig. 12B). This normal thickening is a necessary part of the reproductive process. Figures $12 \mathrm{~A}$ to $\mathrm{C}$ illustrate the changes in the endometrium over the normal cycle. Failure of thickening to occur can be associated with amenorrhea caused by physiological, psychological and/or pharmacology problems. Excessive thickening can also occur. This can result from hormonal imbalances, fibroids, polyps, endometrial hyperplasia and cancer. ${ }^{1}$ Failure of the endometrium to thicken appropriately (either inadequate or excessive thickening) can signal a pathological condition. This can range from delaying pregnancy to life-threatening cancers.

During the secretory phase that follows ovulation, estrogen and progesterone released from the corpus luteum (Figs 13 and 14) continue to produce thickening and swelling of the endometrium (Fig. 12C). During this phase there is a significant increase of glycogen, acid phosphatase and lipids in the endometrium. The histological pattern of such secretory endometrium includes remarkably increased vascularization, which is necessary for delivery of the nutrients and preparation for the fertilized ovum (Fig. 15).

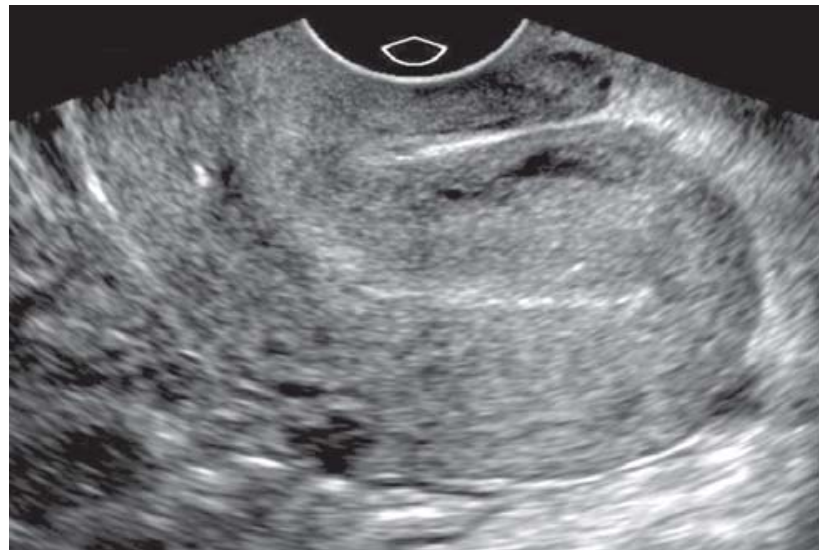

Fig. 12A: Transvaginal scan of the postmenstrual endometrium, which is thin and hyperechogenic

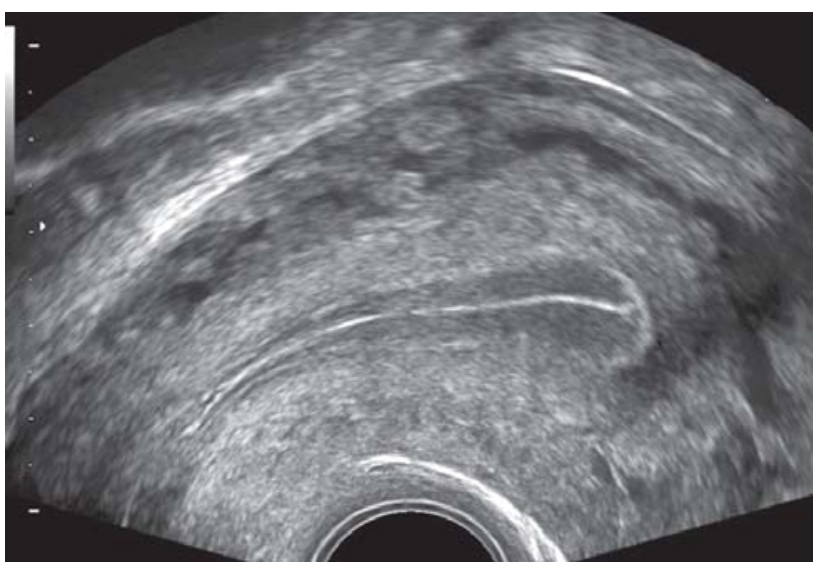

Fig. 12B: Transvaginal scan of the proliferative endometrium. Note triple line appearance of the endometrial lining

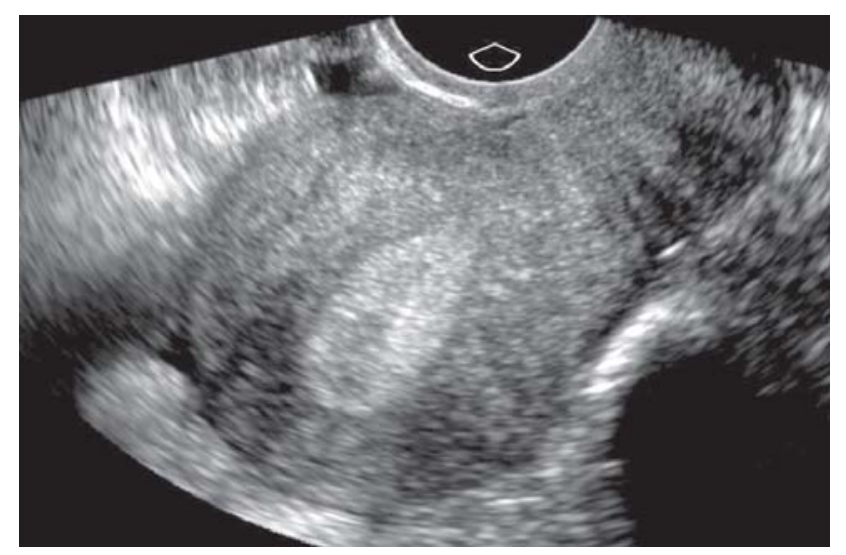

Fig. 12C: Transvaginal scan of the secretory endometrium, which is thick and hyperechogenic

If implantation of the ovum is to occur, it will be within 7-9 days after ovulation. At this time the trophoblastic cells on the surface of the ovum will start to break down the endometrium, releasing abundant amounts of nutrients for the embryo. If implantation does not occur, the corpus luteum decreases its production of estrogen and 


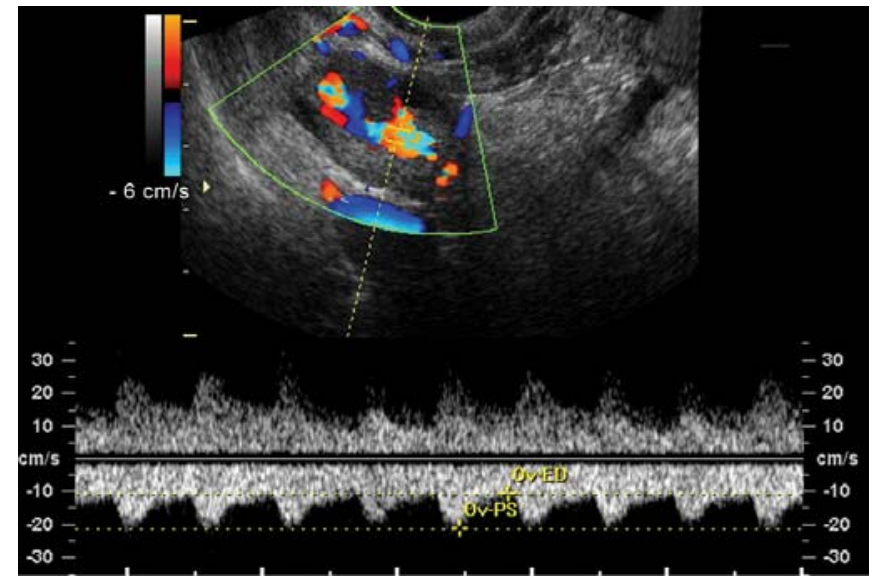

Fig. 13: Transvaginal color Doppler scan of the corpus luteum. A high blood flow velocity and low vascular resistance (RI 0.50$)$ represent typical flow pattern of a mature corpus luteum

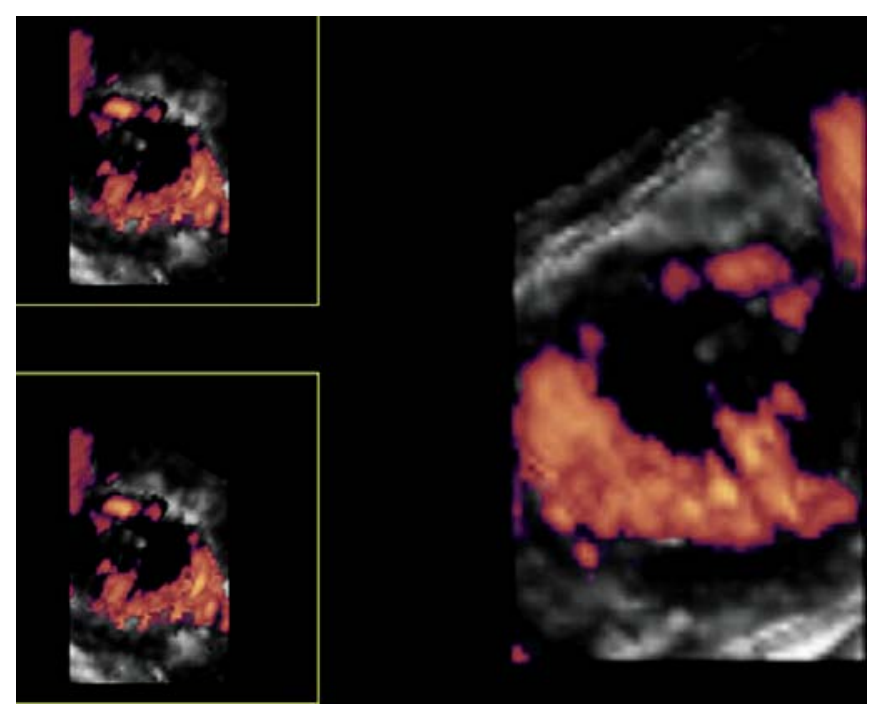

Fig. 14: Three-dimensional power Doppler image of increased ovarian vascularity during the luteal phase

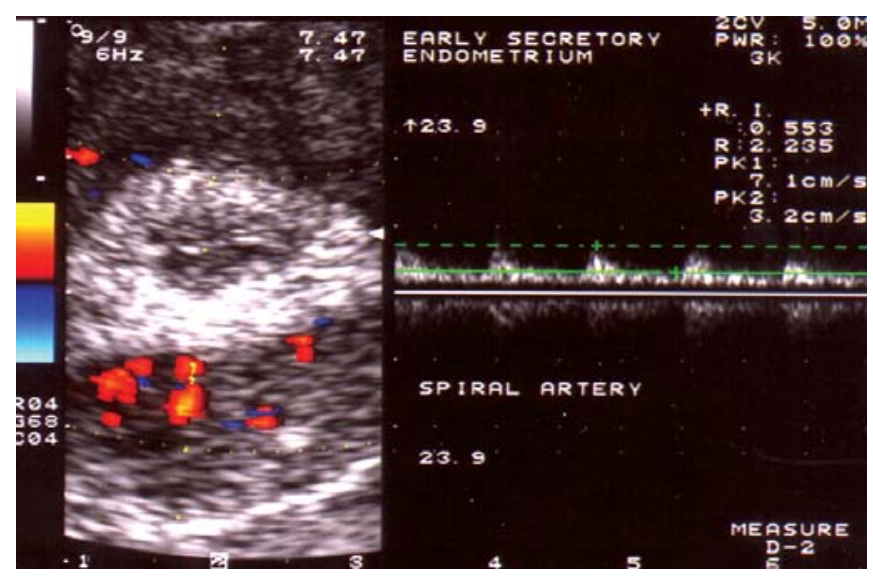

Fig. 15: Transvaginal color Doppler scan of the secretory changed endometrium. Note the color coded area at the periphery of the endometrium, representing spiral arteries. The pulsed Doppler waveform analysis shows low-to-moderate vascular resistance (RI 0.55 ) during the mid-luteal phase of the menstrual cycle progesterone. The lack of hormonal support results in demise of the thickened endometrium and sloughing commences. Within the first 24 hours, the blood vessels in the endometrium become vasospastic, the tissue becomes necrotic, and the endometrium decreases to about $65 \%$ of its original thickness. The discharged fluid will contain about $40 \mathrm{ml}$ of blood and another $35 \mathrm{ml}$ of serous fluid. ${ }^{1}$ Normal clotting does not occur due to the presence of fibrinolysins released along with the discharge. If the volume of the discharge is excessive, clots in the menstrual fluid can appear due to an inadequate amount of anticoagulant factors.

\section{CONCLUSION}

Ultrasound provides safe, noninvasive images of the normal female reproductive structures. These images have provided baseline information useful in identifying abnormalities in both reproductive structures and in alterations in endometrial development. Improved ultrasound techniques now provide 3-D, duplex Doppler, and 4-D images. When compared to the B-mode images and hand-held Doppler techniques of yester year, the change is staggering. The development of newer probes and software has improved clarity of the images while providing analysis of movement and simultaneous evaluation of blood flow. The cost of an evaluation is also substantially below that of other imaging techniques such as CT or MRI. This reduced cost coupled with the more portable size of the ultrasound makes it more available in rural areas and in developing countries. Undoubtedly, the use of ultrasound in the evaluation of female reproduction will continue to be accepted as a useful tool for the obstetrician and gynecologist.

\section{REFERENCES}

1. Guyton AC, Hall JE. Text book of Medical Physiology (11th ed). Elsevier: Philadelphia 2006;81:1011-26.

2. Wilson RC, Kesner JS, Kaufman JM, Uemura T, Akema T, Knobil E. Central electrophysiologic correlates of pulsatile luteinizing hormone secretion in the rhesus monkey. Neuroendocrinology 1984;39(3):256-60.

3. Speroff L, Glass RH, Kase NG. Clinical gynecologic endocrinology and infertility (6th ed). Lippincott Williams and Wilkins: Baltimore 1999;5:168.

4. Belchetz PE, Plant TM, Nakai Y, Keogh EJ, Knobil E. Hypophysial responses to continuous and intermittent delivery of hypothalamic gonadotropin-releasing hormone. Science 1978;202(4368)631-33.

5. Koppen BM and Stanton BA. Berne and Levy Physiology (6th ed). Elsevier: Philadelphia 2008;40.

6. Speroff L, Glass RH, Kase NG. Clinical gynecologic endocrinology and infertility (6th ed). Lippincott Williams and Wilkins: Baltimore 1999;6:210-15.

7. Bulun SE, Adashi EY. The physiology and pathophysiology of the female reproductive axis, in Kronenberg: Williams Textbook of Endocrinology (11th ed). Sanders: New York, 2008;16. 